\title{
MODELAGEM E SIMULAÇÃO DE AQUECEDOR SOLAR VISANDO AQUECIMENTO DE ÁGUA
}

\author{
S. R. TAVARES ${ }^{1}$ e N. G. SOUSA ${ }^{1}$ \\ 1,2Universidade Federal do Triângulo Mineiro, Departamento de Engenharia Química \\ 2E-mail para contato: nadiagsousa@gmail.com
}

\begin{abstract}
RESUMO - O crescente aumento na demanda energética mundial é um dos grandes desafios da atualidade. Sabe-se que o uso da energia elétrica para aquecimento de água é um dos maiores responsáveis pelo alto consumo de energia elétrica no país. Com isto em mente sempre se tenta arrumar alternativas para uma diminuição do uso de energia elétrica. Neste trabalho é possível notar a viabilidade de um aquecedor solar onde utilizando equações foi possível se conseguir um bom rendimento para o aquecedor solar. Dentre as variáveis que foram relevantes para este processo está a temperatura ambiente, que tem uma significante diferença quando tratada como a média do dia e quando considerada durante as vinte e quatro horas do dia.
\end{abstract}

\section{INTRODUÇÃO}

O crescente aumento na demanda energética mundial é um dos grandes desafios da atualidade. Sabe-se que o uso da energia elétrica para aquecimento de água é um dos maiores responsáveis pelo alto consumo de energia elétrica no país. Para reduzir esse consumo, uma alternativa viável é popularizar a utilização da energia solar para tal aquecimento. O Brasil é privilegiado em termos de radiação solar. Segundo a ANEEL, o Plano Nacional de Energia 2030 reproduz dados do Atlas Solarimétrico do Brasil e registra que essa radiação varia de 8 a $22 \mathrm{MJ} / \mathrm{m}^{2}$ durante o dia, sendo que as menores variações ocorrem nos meses de maio a julho, variando de 8 a $18 \mathrm{MJ} / \mathrm{m}^{2}$.

Os primeiros aquecedores surgiram na década de 70, porém somente na década de 90 eles se tiveram um aumento substancial e com isso começaram a ter maiores variações de modelo, qualidade e assim maiores aplicações. Albuquerque et al. (2002) propuseram a análise de como alguns fatores, como a velocidade do vento, influenciam na eficiência de um sistema de aquecimento de água com coletor solar. Eles concluíram que nas condições e no local onde foram feitas as medidas o uso de coletores solares é viável e no verão a radiação solar é fator determinante no sistema.

Kicsiny et al. (2013) propuseram modelos de EDO's estendidas para modelar uma grande espécie de sistemas de aquecimento solar com tubos. Os modelos estendidos são mais razoáveis que os modelos básicos levando em conta a precisão da modelagem de armazenamento, o que é indispensável na previsão e no desenvolvimento da eficiência do sistema de aquecimento solar para o beneficio do consumidor. 
O objetivo deste projeto é a análise e a simulação matemática de um aquecedor solar para sistema de aquecimento de água. Para tal, tem-se como base trabalhos encontrados na literatura, para efeito de comparação e viabilidade do projeto. A utilização de sistemas de aquecimento solar visa diminuição dos custos de energias elétricas além de ser uma fonte de energia renovável e limpa. Os dados de irradiação e temperatura ambiente foram coletados para a cidade de Uberlândia, localizada no Triângulo Mineiro.

\section{DESCRIÇÃO DO PROCESSO}

Um sistema solar de aquecimento de água é composto basicamente por: reservatório de água fria, reservatório térmico, coletor solar e rede de consumo, como mostrado na Figura 1.

Figura 1- Aquecedor Solar (adaptado de Kicsiny et al., 2013)).

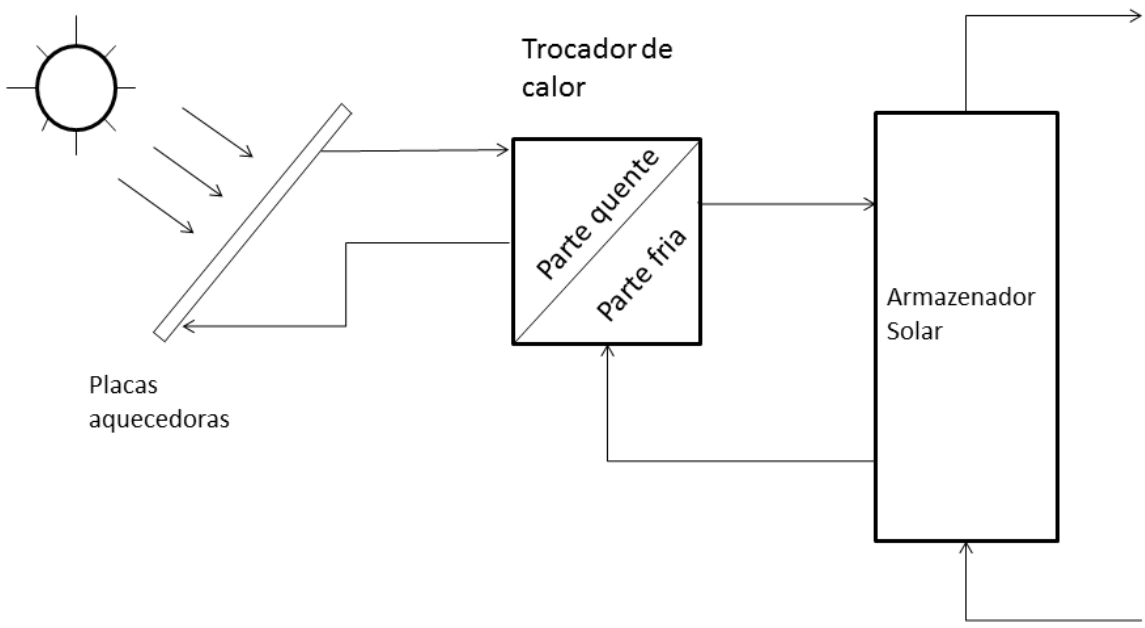

Este trabalho possui natureza teórico-computacional e utilizará o software livre Scilab ${ }^{\circledR}$, para realizar as simulações computacionais dos sistemas estudados.

\subsection{Modelo matemático}

Utilizando as equações de Kicsiny et al. (2013), foi possível um estudo para a região do Triângulo Mineiro. As equações propostas por eles que utilizamos foram:

$$
\begin{aligned}
& \dot{T}_{c}=\frac{A_{c} \eta_{0}}{\rho_{c} c_{c} V_{c}} I_{c}+\frac{U_{L b} A_{c}}{\rho_{c} c_{c} V_{c}}\left(T_{c a}-T_{c}\right)+\frac{v_{c}}{V_{c}}\left(T_{h h}-T_{c}\right) \\
& \dot{T}_{h h}=\frac{\rho_{c} c_{c} V_{c}}{\frac{c_{h} m_{h}}{2}+\rho_{c} c_{c} \frac{V_{h}}{2}}\left(T_{c}-T_{h h}\right)+\frac{\varepsilon k_{h} A_{h}}{\frac{c_{h} m_{h}}{2}+\rho_{c} c_{c} \frac{V_{h}}{2}}\left(T_{h c}-T_{h h}\right)+ \\
& \quad+\frac{\frac{A_{a} k_{a}}{2}}{\frac{c_{h} m_{h}}{2}+\rho_{c} c_{c} \frac{V_{h}}{2}}\left(T_{h a}-T_{h h}\right)
\end{aligned}
$$




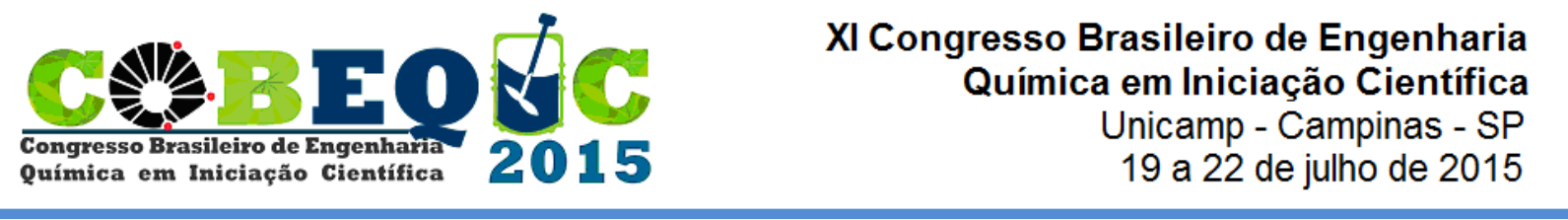

$$
\begin{aligned}
& T_{h c}=\frac{\rho_{s} c_{s} V_{s}}{\frac{c_{h} m_{h}}{2}+\rho_{s} c_{s} \frac{V_{h}}{2}}\left(T_{s}-T_{h c}\right)+\frac{\varepsilon k_{h} A_{h}}{\frac{c_{h} m_{h}}{2}+\rho_{s} c_{s} \frac{V_{h}}{2}}\left(T_{h h}-T_{h c}\right)+ \\
& +\frac{\frac{A_{a} k_{a}}{2}}{\frac{c_{h} m_{h}}{2}+\rho_{s} c_{s} \frac{V_{h}}{2}}\left(T_{h a}-T_{h c}\right) \\
& \dot{T}_{s}=\frac{\mathrm{v}_{l}}{V_{s}}\left(T_{d}-T_{s}\right)+\frac{\mathrm{v}_{s}}{V_{s}}\left(T_{h c}-T_{s}\right)+\frac{A_{s} k_{s}}{\rho_{s} c_{s} V_{s}}\left(T_{s a}-T_{s}\right)
\end{aligned}
$$

em que: $\mathrm{T}_{\mathrm{c}}$ é a temperatura do coletor solar; $\mathrm{T}_{\mathrm{hh}}$ é a temperatura da parte quente do trocador de calor. $\mathrm{T}_{\text {hc }}$ é a temperatura da parte fria do trocador de calor e $\mathrm{T}_{\mathrm{s}}$ é a temperatura do tanque de aquecimento.

\section{RESULTADOS E DISCUSSÕES}

Os parâmetros utilizados para a simulação do modelo estão dispostos na Tabela 1 .

Tabela 1 - Parâmetros da simulação.

\begin{tabular}{|c|c|c|c|c|}
\hline$A_{c=33,3 \mathrm{~m}^{2}}$ & $A_{a=0,24 \mathrm{~m}^{2}}$ & $\mathrm{v}_{c=0,000272 \mathrm{~m}^{3} / \mathrm{s}}$ & $V_{h=0,005 \mathrm{~m}^{3}}$ & $V_{s=2 \mathrm{~m}^{3}}$ \\
\hline$\rho_{s=1000 \mathrm{~kg} / \mathrm{m}^{3}}$ & $c_{s=4200 \mathrm{~J} / \mathrm{kg}}$ & $c_{c=3623 \mathrm{~J} / \mathrm{kgK}}$ & $k_{a=0 \mathrm{~W} / \mathrm{m}^{2} \mathrm{~K}}$ & $A_{s}=4 \mathrm{~m}^{2}$ \\
\hline$U_{L b=7,3 \mathrm{~W} / \mathrm{m}^{2} \mathrm{~K}}$ & $V_{c=0,027 \mathrm{~m}^{3}}$ & $c_{h=464,8 \mathrm{~J} / \mathrm{kgK}}$ & $\varepsilon k_{h=2461,5 \mathrm{~W} / \mathrm{m}^{2} \mathrm{~K}}$ & $A_{h=2 \mathrm{~m}^{2}}$ \\
\hline$\rho_{c=1034 \mathrm{~kg} / \mathrm{m}^{3}}$ & $\eta_{0=0,74}$ & $\mathrm{v}_{l=0,0001 \mathrm{~m}^{3} / \mathrm{s}}$ & $v_{s}=0,00175 \mathrm{~m}^{3} / \mathrm{s}$ & $m_{h=37 \mathrm{~kg}}$ \\
\hline$T_{h a=298,15 \mathrm{~K}}$ & $T_{d=298,15 \mathrm{~K}}$ & $k_{s=1 \mathrm{~W} / \mathrm{m}^{2} \mathrm{~K}}$ & $T_{s a=298,15 \mathrm{~K}}$ & \\
\hline
\end{tabular}

Com as Equações 1 a 4 e os valores de irradiação e de temperatura ambiente, obtidos através do site do INMET (Instituto Nacional de Meteorologia), foi possível uma comparação utilizando a temperatura média e a temperatura durante as 24 horas, ou seja, variando ao longo do dia. A fim de comprar os resultado simulados a eficiência média será calcula pela Equação 5.

$$
\eta=\frac{v_{s} \rho_{s} c_{s}\left(T_{s \text { média }}-T_{s a}\right)}{A_{c} I_{c \text { médio }}}
$$

Os resultados da simulação são mostrados nas Figuras 2 e 3. 
Figura 2 - Comportamento para as temperaturas do coletor solar e da parte quente do trocador ( - $T_{c a}$ constante; -.- $T_{c a}$ variando).
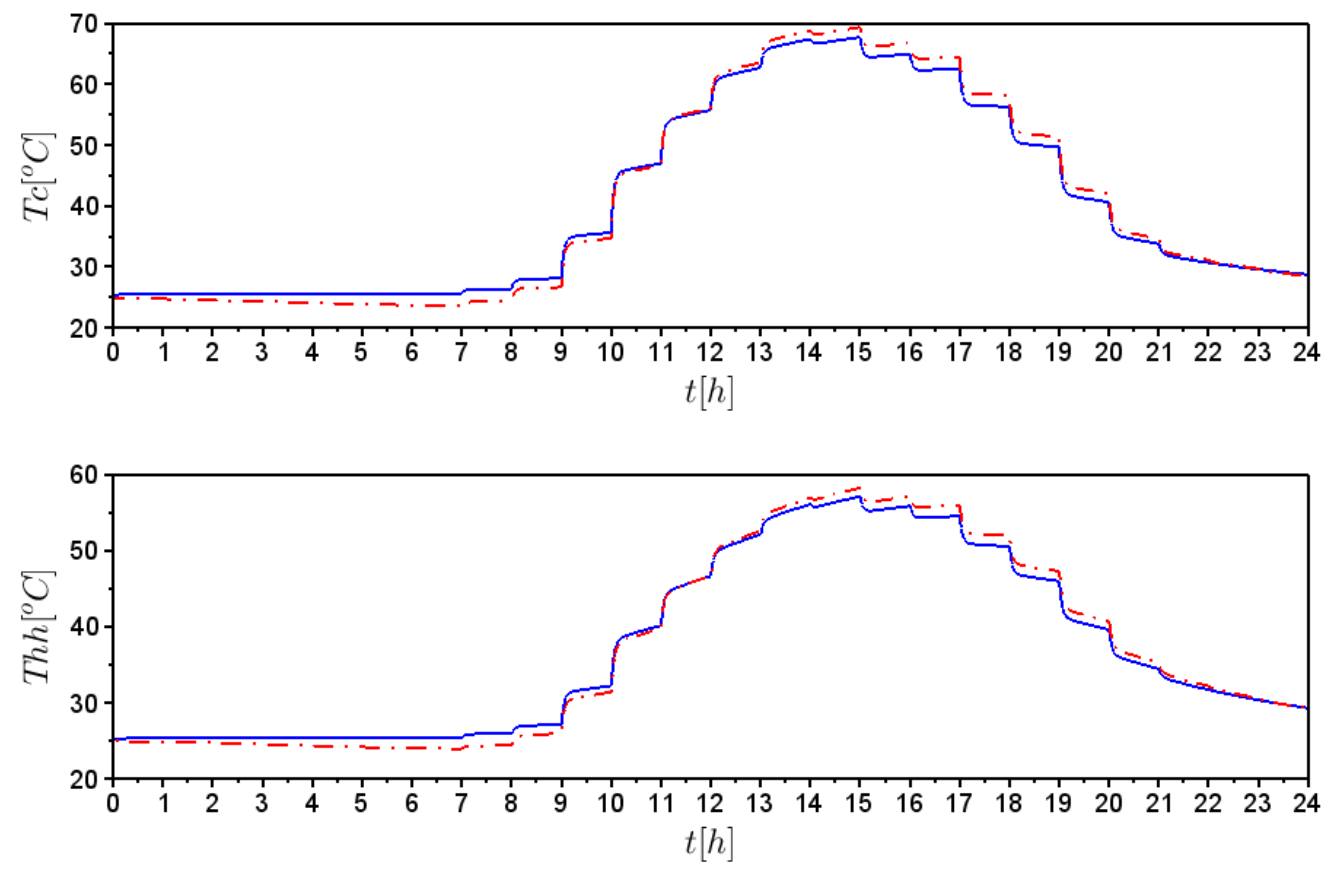

Figura 3 - Comportamento para as temperaturas da parte fria do trocador e do armazenador ( $-T_{c a}$ constante; -.- $T_{c a}$ variando) .
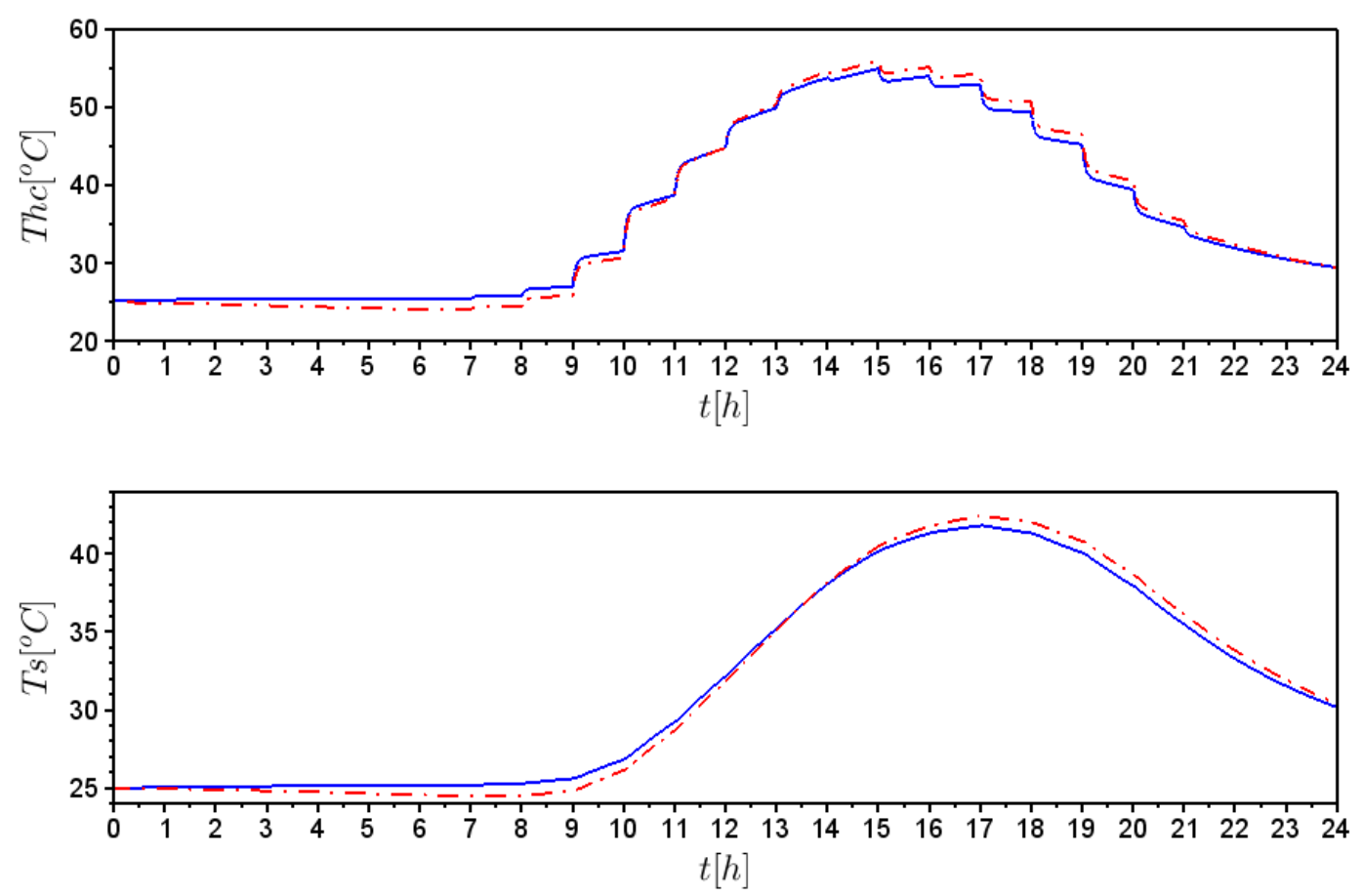

Analisando os resultados obtidos, pode-se notar que a influência da temperatura ambiente é pouco significativa. Entretanto, ao analisar a eficiência percebe-se que ao 
considerar a temperatura variando ao longo do dia tem-se $\eta=48 \%$ e a eficiência considerando a média das temperaturas é de $\eta=30,5 \%$. Isso ocorre pelo fato de que ao considerarmos hora a hora temos um modelo com maior grau de precisão do que quando fazemos a média.

\section{CONCLUSÕES}

As simulações efetuadas mostraram que as unidades de aquecimento solar de água são ferramentas em potencial para serem utilizadas como alternativas para reduzir o consumo de energia, pois, apresentaram eficiências próximas de 50\%. Entretanto é necessário levar em conta a influência de algumas variáveis do processo, como a variação da temperatura ambiente.

Os resultados obtidos quando foram implementados os dados coletados para a região do Triângulo Mineiro pôde-se notar que quando se utiliza a temperatura variando ao longo do dia tem-se temperaturas máximas o que leva a uma maior absorção de energia solar pelo coletor e assim influencia diretamente as demais temperaturas do processo $(\eta=48 \%)$. Isso não é notado quando se utiliza valores médios para a temperatura ambiente, neste caso as máximas não serão atingidas reduzindo assim eficiência do sistema de aquecimento solar $(\eta=30,5 \%$. $)$.

\section{NOMENCLATURA}

$A_{a}=$ área da superfície do trocador de calor para o ambiente $\left(\mathrm{m}^{2}\right)$

$A_{c}=$ área de superfície do coletor no campo $\left(\mathrm{m}^{2}\right)$

$A_{h=}$ área da superfície de transferência de calor dentro do trocador $\left(\mathrm{m}^{2}\right)$

$A_{s}=$ área superficial externa do armazenador solar $\left(\mathrm{m}^{2}\right)$

$c_{c}=$ capacidade do calor específico do fluido coletor $(\mathrm{J} / \mathrm{kg} \mathrm{K})$

$c_{h}=$ capacidade de calor específico do material do trocador de calor $(\mathrm{J} / \mathrm{kg} \mathrm{K})$

$c_{s}=$ capacidade do calor específico da água $(\mathrm{J} / \mathrm{kg} \mathrm{K})$

$\varepsilon k_{h}=$ coeficiente de transferência de calor dentro do trocador $\left(\mathrm{W} / \mathrm{m}^{2} \mathrm{~K}\right)$

$I_{c}=$ irradiação solar global na superfície do coletor $\left(\mathrm{W} / \mathrm{m}^{2}\right)$

$k_{a}=$ coeficiente de perda de calor do trocador para o ambiente $\left(\mathrm{W} / \mathrm{m}^{2} \mathrm{~K}\right)$

$k_{s}=$ coeficiente de perda de calor do armazenador solar para o ambiente $\left(\mathrm{W} / \mathrm{m}^{2} \mathrm{~K}\right)$

$m_{h}=$ massa do trocador de calor vazio $(\mathrm{kg})$

$=$ Temperatura do coletor solar $(\mathrm{K})$

$T_{d}=$ Temperatura da água da torneira $(\mathrm{K})$ 
$T_{h a}=$ Temperatura ambiente do trocador de calor $(\mathrm{K})$

$T_{h c}=$ Temperatura da parte fria do trocador (parte do armazenador) (K)

$T_{h h}=$ Temperatura da parte quente do trocador (parte do coletor) (K)

$T_{s a}=$ Temperatura ambiente do armazenador solar (K)

$=$ volume do coletor solar no campo $\left(\mathrm{m}^{3}\right)$

$V_{h}=$ volume total do trocador de calor $\left(\mathrm{m}^{3}\right)$

$V_{s}=$ volume do armazenador solar $\left(\mathrm{m}^{3}\right)$

\section{REFERÊNCIAS}

ALBUQUERQUE. T. T. de A.; BARCELLOS. K. M.; LYRA. R. F. da F. Influência dos Parâmetros Meteorológicos sobre a Eficiência de um Coletor Solar Plano Destinado a Aquecimento de Água na Região de Maceió-Al. Alagoas, 2002.

KICSINY. R.; NAGY. J.; SZALÓKI. CS. Extended Ordinary Differential Equation Model for Solar Heating Systems with Pipes. Applied Energy, v. 129, p. 166-176, 2013.

OLIVEIRA, A. G. A. DE. Modelagem e Simulação de um Sistema Solar de Aquecimento e Distribuição de Água Quente. Belo Horizonte, 2014.

INMET, Instituto Nacional de Meteorologia. Disponível em: $<$ http://www.inmet.gov.br/portal/index.php?r=estacoes/estacoesAutomaticas $>$.

KICSINY. R.; VARGA. Z. Real-time state observer design for solar thermal heating systems. Applied Mathematics and Computation, v. 218, p. 11558-11569, 2012. 